Schrankenproblematik der Grundrechte in der koreanischen Verfassung

Ein Bericht, erstattet anläßlich des Symposiums, 100 Jahre koreanisch-deutsche Beziehungen in der Rechtswissenschaft, am 21.-22. September 1984 in Seoul

Von Young Huh

\title{
I. Einleitung
}

Ich soll über die Schranken der Grundrechte in der Koreanischen Verfassung sprechen. Ich muß mich jedoch darauf beschränken, die Grundlinien der Schrankenregelung der koreanischen Verfassung zu skizzieren und darüber mein Urteil abzugeben. Ich möchte daher auch nur diejenigen Aspekte ansprechen, die zur Abgrenzung der Thematik unerläßlich sind. Von besonderer Bedeutung ist dabei die Wertigkeit der Grundrechte im Spannungsfeld von Bürger und Staat.

Die Frage nach der Bedeutung und nach dem Inhalt staatsbürgerlicher Grundrechte, ihrer Bewahrung und ihrer Verteidigung sind mit Gedanken verbunden, die zum Bereich der Menschenrechtsidee gehören und die daher bei jeder bedeutenden Wendung in der wissenschaftlichen und politischen Geschichte der neueren Zeit erneut die Aufmerksamkeit auf sich ziehen. Das allein wäre Grund genug, die Schrankenproblematik der Grundrechte auf die Tagesordnung eines Symposioms zu setzen, an dem Rechtswissenschaftler aus Deutschland und Korea beteiligt sind. In einem solchen Kreise jedoch haben die Probleme der Menschenrechte auch eine besondere Bedeutung, die aus der geschichtlich begründeten Besonderheit in der Relation zwischen Deutschland und Korea hervorgeht. Das gab der Entscheidung der Koreanisch-Deutschen Gesellschaft für Rechtswissenschaft ihr eigenes Gewicht, die Menschenrechte zum zentralen Gegenstand dieses Symposions zu machen. Ich hatte dabei die Aufgabe, in gegenseitiger Absprache mit Herrn Isensee Themen zu formulieren und die Themen soweit wie möglich aufeinander abzustimmen.

So also kann ich nunmehr nur in der Hoffnung, auch seine Intentionen richtig zu interpretieren, etwas über die Schrankenproblematik der Grundrechte sagen, was primär bei uns in Korea gilt.

Bi-nationale Aspekte und rechtsvergleichende Sicht böten sich dabei an, weil eine bi-nationale Rechtsvergleichung gerade das Charakteristikum dieses Deutsch-Koreanischen Symposions bildet.

Rechtsvergleichende Bezüge würden sich jedoch in unserem Themengebiet eher als unfruchtbar erweisen. Die Gewährleistung der Grundrechte und insbesondere die Schranken der Grundrechte sind in besonderem Maße national bedingt und hängen mit histori- 
schen, sozialen und politischen Gegebenheiten der jeweiligen politischen Einheit aufs engste zusammen. Eine rechtsvergleichende Untersuchung verspricht deshalb nur begrenzte Erkenntnis.

Immerhin sei auf den Umstand hingewiesen, daß sowohl das Grundgesetz der Bundesrepublik Deutschland als auch die Verfassung der Republik Korea die Grundrechte garantieren und zugleich in Gestalt von Gesetzesvorbehalt auf die Schranken der Grundrechte hinweisen. Man sollte sich jedoch nicht darüber hinwegtäuschen, daß eine verfassungsrechtliche Garantie der Menschenrechte keinen klaren Indizwert für Niveau und Dichte der Grundrechtspolitik eines Landes hat. Diese Feststellung ist besonders aufschlußreich, wenn man sich der Tatsache bewußt wird, daß einerseits sich ein Grundrechtskata$\log$ in den Verfassungen der kommunistischen Länder ebenso vorfindet wie etwa in der Volksrepublik China und in der Sowjetunion, um nur die sogenannten "Muster-Staaten" des Kommunismus zu nennen, und daß andererseits sich ein Grundrechtskatalog offenbar in totalitären Staaten besonderer Beliebtheit erfreut. Der Umstand, daß es keinen einzigen Staaten auf der Welt gibt, der einen Grundrechtskatalog in seine Verfassung nicht aufgenommen hat, bestätigt auch die tendenzielle Irrelevanz eines Grundrechtskatalogs. Anders gewendet: $\mathrm{Ob}$ ein Staat tatsächlich auf die Menschenrechte achtet, hängt nicht von der Existenz eines verfassungsrechtlichen Grundrechtskagalogs $\mathrm{ab}$.

Ferner setzen etwaige Grundrechtseinschränkungen die Existenz einer Eingriffsvorbehaltsklausel auch nicht immer voraus, wie etwa die Institution der Schranken der vorbehaltlosen Grundrechte zeigt. Darüber hat uns Herr Isensee eben ausführlich berichtet.

Seinem Vortrag ist zu entnehmen, wenn ich ihn richtig verstanden habe, daß Schranken der vorbehaltlosen Grundrechte in der Bundesrepublik Deutschland in erster Linie bei den liberalen Status-negativus-Grundrechten, also etwa der Religions-, Gewissens- und Kunstfreiheit erörtert werden, und daß aber das Thema sich auch bei den Status-positivus-Grundrechten, etwa dem Teilhabe-Grundrecht auf einen Studienplatz, und sogar bei den Status-activus-Grundrechten, etwa der Gleichheit der Wahl, stellt.

\section{Das Problem}

Daran erkennt man, daß das Schrankenproblem der Grundrechte ein vielschichtiges ist. Es wirft nämlich viele Fragen in die Debatte: Ob und inwiefern dürfen die Grundrechte eingeschränkt werden? Wann ist die Einschränkung der Grundrechte überhaupt zulässig? Sollen die Schranken der Grundrechte allein durch das Gesetz bestimmt werden? Bezeichnet die Institution des Gesetzesvorbehalts Stärke oder/und Schwäche der Grundrechte? Sind immanente Grenzen der Grundrechte Form oder Voraussetzung von Grundrechtseinschränkungen?

Diese Fragen, die sich vermehren ließen, zeigen zur Genüge, wie vielfältig das Schrankenproblem auftauchen kann, und wie nötig und schwierig es ist, die Schrankenprobleme der Grundrechte befriedigend zu lösen. Die in den Lösungsversuchen gesammelten 
Erfahrungen eines demokratischen Staates erteilen auch für andere demokratische Länder wichtige Lehren. Wir alle wissen aber, wie tastend und wie problembeladen alle Versuche sind, diese Fragen im innerstaatlichen Bereich eindeutig zu beantworten.

Die Frage nach den Schranken der Grundrechte steht gewiß in einem engen Zusammenhang mit der Grundrechtstheorie, und die Beantwortung dieser Fragen kommt auch auf den rechts- und staatsphilosophischen Standpunkt an, auf den man sich stellt. Die Schranken der Grundrechte sind andererseits nicht über die Schrankenregelung des jeweiligen Verfassungsrechts weg zu ermitteln.

\section{Stellungnahmen zur Schrankenregelung der koreanischen Verfassung}

Der Grundrechtskatalog der koreanischen Verfassung endet mit der Bestimmung: "Alle Grundrechte können durch Gesetz eingeschränkt werden, sofern es für die Staatssicherheit, die Aufrechterhaltung der Ordnung oder das Gemeinwohl erforderlich ist. Jedoch ist nicht zulässig, ein Grundrecht in seinem Wesensgehalt anzutasten" (Art. 35 Abs. 2).

Die Freiheit und Rechte des Staatsbürgers stehen somit ohne Ausnahme unter einem Gesetzesvorbehalt. Die sogenannten absoluten Grundrechte, die auch durch Gesetz nicht eingeschränkt werden dürfen, kennt die koreanische Verfassung nicht. Die Schrankenproblematik der vorbehaltlosen Grundrechte ist infolgedessen nur ein Gegenstand reintheoretischer Erörterungen ohne praktische Bedeutung. Denn die Limitierungen sind bei allen Grundrechten gleich: System der abgestuften Gesetzesvorbehalte grundgesetzlicher Prägung ist der koreanischen Verfassung unbekannt. Es gibt keine spezifischere Technik, bei der die jeweiligen Grenzen jedes einzelnen Grundrechts gesondert festgelegt werden. Der Schrankenregelung der koreanischen Verfassung liegt, wie mir scheint, keine einheitliche Konzeption zugrunde. Sie leidet vielmehr an Ungereimtheit und Widersprüchlichkeit. Wenn z. B. die sog. "Staatssicherheit" als einen Einschränkungsgrund anerkannt wird und wenn sie schon einen Grundrechtseingriff rechtfertigen sollte, ist man eine einleuchtende Erklärung dafür nicht abzugeben, warum die Staatssicherheit der Wesensgehaltsgarantie preisgegeben werden soll. Ebensowenig läßt sich kein vernünftiger Grund dafür erkennen, daß nach Art. 35 Abs. 2 der koreanischen Verfassung die liberalen Status-negativus-Grundrechte gleichermaßen wie die Status-positivusGrundrechte und wie die Status-activus-Grundrechte ihre Schranken in den Gesetzesvorschriften finden und nur nach Maßgabe der Gesetze gelten.

Ich will nun versuchen, Schranken, die die koreanische Verfassung den Grundrechten zieht, auf einige Prinzipien zu reduzieren und die der Schrankenregelung zugrunde liegenden Gedanken ans Licht zu bringen. Wenn heute in der Bundesrepublik Deutschland die totale Grundrechtsbindung der gesetzgebenden Gewalt im Grundgesetz ausdrücklich garantiert ist, da kann das Gesetz prinzipiell nur Garant der Freiheit, aber nicht ihr Gegner sein. Wo aber, wie in der Republik Korea, eine Verfassung im Banne der Ideen Rousseaus und in der dieser Ideologie verhafteten Grundrechtstradition dezisionistischer Provenienz steht, da ist kein Raum für den Gedanken, daß die Grundrechte auch den 
Gesetzgeber binden und beschränken könnten oder gar sollten. Dort erschöpft sich also die Bedeutung der Grundrechte in der Garantie gesetzmäßiger Verwaltung und Justiz, im Vorrang und Vorbehalt des Gesetzes. Die Institution des Gesetzesvorbehaltes bezeichnet in der koreanischen Verfassung halt Schwäche der Grundrechte. Schwäche bewirkt der Gesetzesvorbehalt dadurch, daß er die Grundrechte dem Ein- und Zugriff des Gesetzes öffnet. Er schützt sie also nur vor gesetzwidrigen oder gesetzlosen Eingriffen der Verwaltung und Justiz, während er sie zur Disposition der Legislative stellt. Daß diese ihre Dispositionsfreiheit mißbrauchen kann, daß auch und gerade die Legislative die Freiheit des Bürgers bedrohen, beschränken und vernichten kann, und zwar auch dann, ja gerade dann, wenn sie in der Hand der Volksvertretung liegt, das haben die Deutschen nach bitteren Erfahrungen mit der Allmacht eines "demokratischen" Gesetzgebers nationalsozialistischer Prägung begriffen; die Koreaner aber haben das auch bei der Gründung ihrer fünften Republik im Jahre 1980 nicht gewußt. Ihre Verfassungsväter, die nicht Montesquieu und Rudolf Smend, sondern Rousseau und Carl Schmitt zu ihren "Chefideologen" erkoren hatten, verstanden die Grundrechte in erster Linie als Schranken der Verwaltung und Justiz.

So wird die Frage nach der Zulässigkeit von Grundrechtseinschränkungen, wenn ich recht sehe, jedenfalls in der Praxis und Rechtsprechung nicht als problematisch angesehen, sondern schlicht und einfach dahin beantwortet, daß die Schranken der Grundrechte durch das Gesetz bestimmt werden. Diese schlichte Beantwortung beruht primär auf dem Gedanken, wo das Gesetz der Ausdruck des allgemeinen Willens ist, an dessen Formulierung alle Staatsbürger durch ihre Repräsentanten mitzuwirken befugt sind, da können Gesetz und Bürgerfreiheit nicht in Konflikt geraten.

$\mathrm{Da}$ die koreanische Verfassung normiert, zu welchen Zwecken, in welchem Umfang und in welchen Formen ein Grundrecht eingeschränkt oder in ein Grundrecht eingegriffen werden darf, mag den Anschein erwecken, als ob es sich um eine rechtsstaatliche Regelung handelte. Aber bei Lichte besehen ist man nicht zu übersehen, daß Begriffe, wie die "Staatssicherheit", "Aufrechterhaltung der Ordnung" und das "Gemeinwohl" keine effektiven, rechtsstaatlichen Limitierungen für Gesetzesvorbehalte sein zu können. Sie sind viel zu unbestimmt und vieldeutig, um überhaupt mit einem rechtsstaatlichen Lorbeer bekränzt zu werden.

Um so größere Bedeutung kommt der Wesensgehaltsgarantie der Grundrechte zu. Die koreanische Verfassung zieht eine allgemeine Schrankenschranke in dem Satz, daß "es nicht zulässig ist, ein Grundrecht in seinem Wesensgehalt anzutasten" (Art. 35 Abs. 2 Satz 2). Dieser Satz scheint immerhin eine Grenze für alle staatlichen Grundrechtseingriffe zu setzen. Dennoch ist nicht zu leugnen, daß die Grundrechte in Korea trotz einer Wesensgehaltsgarantie nach Maßgabe der Gesetze gelten.

Die Wesensgehaltsgarantie, die mit Blickrichtung auf die einem ausdrücklichen Gesetzesvorbehalt unterstehenden Grundrechte zumindest den Wesensgehalt dieser Rechte als unantastbar erklärt, dürfte rechtlich kaum abgesichert sein. Denn rechtlich kaum abzusichern sind die Modalitäten der Interpretation. Die Art und Weise etwa, in der die der Konkretisierung der Wesensgehaltsklausel dienende Verhältnismäßigkeitsprüfung vor- 
genommen wird, ist nur in den Umrissen präformierbar und richtet sich nicht zuletzt nach den Oszillationen der jeweiligen politischen Kultur, dem Bewußtsein der interpretierenden Richter und ihrer "Umstände" im rechtshistorischen Sinne des Wortes.

Im Extremfall einer Entartung der Wesensgehaltsgarantie als des letzten Garanten der verfassungsrechtlichen Grundrechte, wenn also - um Friedrich Schiller zu zitieren - "der Bedrückte nirgends Recht kann finden", mag er unter Berufung auf Naturrecht zum Widerstandsrecht seine Zuflucht nehmen.

Die heutige koreanische Staatsrechtslehre ist aber gegenüber der Vorstellung einer überstaatlichen Geltung des Widerstandsrechtes sehr zurückhaltend geworden. Die Auffassung, daß "der Bestand der überstaatlichen Grundrechte von ihrer Formulierung in Gesetzen völlig unabhängig" sei, daß sie "absolut" seien und "durch Verfassungstexte nicht relativiert werden" können, ist in dieser Stringenz kaum mehr anzutreffen. Vorherrschend ist die Úberzeugung, daß der durch die positivierten Grundrechte gewährleistete verfassungsrechtliche Status nicht als bloße Anerkennung eines vorstaatlichen und vorrechtlichen und daher von Staat unabhängigen Status natürlicher Freiheit und Gleichheit verstanden werden könne. "In der Wirklichkeit geschichtlichen menschlichen Lebens sind Freiheit und Gleichheit des einzelnen niemals von Natur aus vorgegeben, sondern sie bestehen nur insoweit, als sie den Bestand des Staates nicht gefährden." Verneint ist damit nicht nur die formale Zweidimensionalität der Grundrechte, die sich einerseits als individuelle Anspruchsnormen und andererseits als legislative und exekutive Handlungs- und judikative Kontrollnormen darstellen, sondern auch die Frage der spezifischen Wertigkeit der Grundrechte.

Wenn in Korea von Grundrechten die Rede ist, so ist zumindest die Assoziation jener von Friedrich Schiller poetisch beschworenen "ewigen Rechte, die droben hangen, unveräußerlich" kaum haltbar. Die Grundrechte, das waren und sind nach dem Verständnis der politischen Führung dieses Landes eben stets jene durch staatliche Satzung aufhebbaren nicht "ewigen Rechte". Dieses Verständnis der Menschenrechte ist auch durch den im 19. und 20. Jahrhundert mehr oder weniger dezidierten Vormarsch rechtspositivistischer Tendenzen nur verstärkt. Und obwohl das Selbstbewußtsein des Rechtspositivismus im Angesicht der Perversionen der faschistischen Epoche eine entscheidende Erschütterung erfahren hatte, ist diese Úberlieferung in Korea immer noch machtvoll emporgetragen worden.

Angesichts der in Korea in vergleichsweise üppiger Weise eingeräumten staatlichen Grundrechtseingriffe und angesichts der in Korea vergleichsweise lückenhafter Weise eingeräumten gerichtlichen Rechtsschutzes gegen legislative und exekutive Akte ist die Effizienz des Grundrechts äußerst fragwürdig. Da die Grenze zwischen einer legitimen Aktualisierung der Verfassungsordnung und einem disruptiven Abrücken von den Prinzipien der Verfassungsordnung erfahrungsgemäß nur in seltenen Extremfällen eindeutig festzustellen ist, ist die Effizienz des Widerstandsrechts ebenfalls äußerst fragwürdig. Die sicherste Gewähr für die Lebenskraft der in der koreanischen Verfassung positivierten Grundrechte ist in der von möglichst vielen Bürgern - und vorab natürlich von den dem Recht verpflichteten Juristen - zu besorgenden Pflege der politischen Kultur unse- 
res Landes zu sehen. Die Schrankenproblematik der Grundrechte - und ebenfalls die damit zusammenhängende Effizienz des Grundrechts - ist weniger eine Frage der Paragraphen als der Pflege der politischen Kultur unseres Staates. Entscheidender ist der Stil und die Glaubwürdigkeit des politischen Systems sowie die Bereitschaft seiner Bürger, für die Grundrechte einzutreten.

Mit diesem Diktum, das zugleich auch als Appell an uns alle dienen mag, möchte ich meinen kurzen Uberblick abschließen.

\section{Darstellungen zur internationalen Politik und Entwicklungspolitik}

Herausgegeben von Prof. Dr. Klaus Jürgen Gantzel, Institut für Internationale Angelegenheiten der Universität Hamburg

\section{Land Reform and Dispute Processing in the Philippines}

The two empirical studies of this volume deal with a special aspect of the land reform in the Philippines, namely the question in which manner conflicts and legal disputes are caused by land reform and where, how, and with which results these conflicts are regulated and decided. The Geman lawyer Ulrich Rausch investigated the conflict processing in agrarian disputes at the institutions set up for this task in two provinces, Nueva Ecija and Pangasinan. The Filipino socio-anthropologist Norma Tinambacan surveyed the agrarian conflicts in two villages in Nueva Ecija and analysed the mode of settlement in either a pre-institutional or institutional framework.

1984, 144 S., kart., 38,- DM

ISBN 3-7890-0978-4

Nomos Verlagsgesellschaft

Postfach $610 \cdot 7570$ Baden-Baden

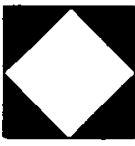

